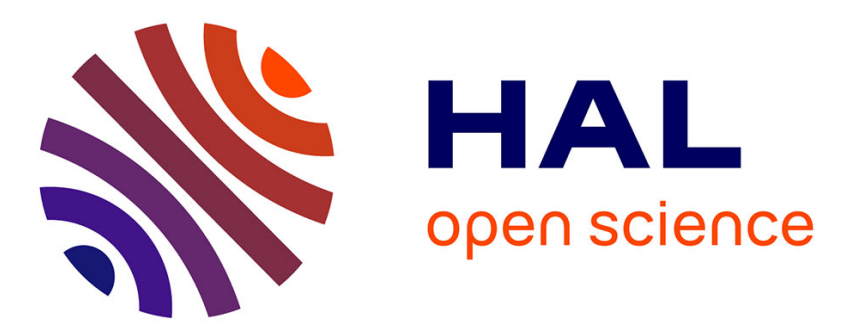

\title{
From constraint satisfactions to periodic positive invariance for discrete-time systems
}

Martin Soyer, Sorin Olaru, Zhou Fang

\section{To cite this version:}

Martin Soyer, Sorin Olaru, Zhou Fang. From constraint satisfactions to periodic positive invariance for discrete-time systems. 2020 59th IEEE Conference on Decision and Control (CDC), Dec 2020, Jeju Island (virtual), South Korea. pp.4547-4552, 10.1109/CDC42340.2020.9304469 . hal-03110392

\section{HAL Id: hal-03110392 \\ https://hal-centralesupelec.archives-ouvertes.fr/hal-03110392}

Submitted on 14 Jan 2021

HAL is a multi-disciplinary open access archive for the deposit and dissemination of scientific research documents, whether they are published or not. The documents may come from teaching and research institutions in France or abroad, or from public or private research centers.
L'archive ouverte pluridisciplinaire HAL, est destinée au dépôt et à la diffusion de documents scientifiques de niveau recherche, publiés ou non, émanant des établissements d'enseignement et de recherche français ou étrangers, des laboratoires publics ou privés. 


\title{
From constraint satisfactions to periodic positive invariance for discrete-time systems
}

\author{
Martin Soyer $^{1,2}$, Sorin Olaru ${ }^{2}$, Zhou Fang $^{1}$
}

\begin{abstract}
This paper deals with the analysis of the trajectories of autonomous dynamical systems with respect to static constraints. Two notions of constraints satisfaction and set-invariance are introduced in order to relax the classical definitions and offer a new perspective with potentially increased flexibility in the topology of the candidate sets. The relaxation comes from the possibility to violate the constraints for intervals of finite length along the evolution of the trajectory. In this line of developments, two alternative definitions emerge: a weak one which allows the validation of each constraint taken independently with an upper bound on the violation interval and the other one which imposes the length of the interval with a guarantee of constraint satisfaction. The main interest of those concepts is to handle simpler sets as positively invariant candidates with clear advantages in constrained control design.
\end{abstract}

\section{INTRODUCTION}

The analysis of constrained dynamical systems has a long history with well established theoretical concepts and well known design tools. On the theoretical side one can recall the mathematical theory of viability [1] and the set theoretic methods [2] covering among other notions the positive invariance. On the applications side, the constrained control and model predictive control in particular are among the design techniques with established popularity [3]. Other topics involving constraints at different stages are the reference governors [4] and the fault detection and isolation [5].

The present work is closely related to the invariant set characterization for constrained dynamical systems. The long line of developments on this topic had an impetus in the 80s and 90s with fundamental results [6]-[8] followed up by extensions and generalization to monotonicity and comparison principles for constrained systems analysis [9].

Often, the system limitations can be modeled as (polyhedral) constraints or alternatively by convex sets and whenever the dynamics can be assimilated to linear systems, well establish computational tools (e.g. the ones leading to the maximal output admissible set [10]) can be employed. However these constructions can be often time consuming and lead to the high complexity representation of the resultant set. Considering approximation of this set may lose the invariance property, and inner invariant approximation computation was seen as a potential alternative [11].

This work explores a novel perspective on the analysis of constraints satisfaction by relaxing the constraint validation

\footnotetext{
1 Renault Technocentre, AD Simulation \& VR center, Guyancourt, France martin. soyer/zhou. fangdrenault.com

2 Université Paris-Saclay, CNRS, CentraleSupélec, Laboratoire des signaux et systèmes, 91190, Gif-sur-Yvette, France martin.soyer/sorin.olaruecentralesupelec.fr
}

to an interval along the trajectories. In other words the trajectories are allowed to violate the constraints as long as the future iterations will come back on the feasible domain and all these constraints violations can be upper bounded in terms of the interval of time. Two notions emerge: a weak version of constraint satisfaction where only an upper bound is imposed on the length of violation window. As a counterpart, the strong satisfaction of constraints on a interval of predefined length, imposes the return to the feasible domain precisely at the end of this lap of time. Using these principles, the set-invariance notions are revisited, establishing the weak and strong periodic invariance (denoted $p$-invariance) of a given set in the state space.

The advantages of dealing with such relaxed notions of constraint satisfaction and invariance can be related to the use of periodic invariant sets in control design which have been shown to bring interesting computational alternatives in [12], [13] and as a benchmark in a practical Driving Simulation test-case [14].

The paper is organized as follows: Section II presents the notion of weak satisfaction of constraints, starting from the trajectories and gradually extending the concepts to multiple constraints and tubes of trajectories. Section III moves the objective to the set invariance and provides the weak notion by focusing on the role of each trajectory in the economy of the violation of the set-membership. Finally, the Section IV provides a link in between the two constructions through the strong counterparts of both constraint satisfaction and positive invariance.

Notations: The power $n \in \mathbb{N}$ of a function is denoted by

$$
f^{n}(x)=\underbrace{f \circ \ldots \circ f}_{n \text { times }}(x)=\underbrace{f(f(f(\ldots)))}_{n \text { times }}(x) .
$$

The subset of integers between $a$ and $b$ is denoted $\mathbb{N}_{[a, b]}=$ $\mathbb{N} \cap[a, b]$. The open ball of radius $r>0$ centered in the origin is denoted $\mathcal{B}_{0}(0, r)$.

$\bar{\Omega}$ denotes the frontier of $\Omega$. $\mathcal{V}(P)$ denotes the set of vertices of a bounded polyhedral set $P$. A convex set $\Omega \subset$ $\mathbb{R}^{n}$ is said to be positively invariant with respect to the autonomous system $x(k+1)=f(x(k))$ if for any initial state $x_{0} \in \Omega$ we have $f\left(x_{0}\right) \in \Omega$.

$$
\mathcal{C}^{0}\left(\mathbb{R}^{n}, \mathbb{R}^{m}\right) \text { denotes the continuous functions from } \mathbb{R}^{n} \text { to }
$$
$\mathbb{R}^{m}$. 


\section{ChARACTERIZATION OF TRAJECTORIES WITH RESPECT TO SUBLEVEL SETS}

\section{A. Preliminary notions and definitions}

Consider a discrete-time autonomous system:

$$
x(k+1)=f(x(k))
$$

defined by a mapping $f \in \mathcal{C}^{0}\left(\mathbb{R}^{n}, \mathbb{R}^{n}\right)$. Given an initial condition $x_{0}$, the forward trajectory of the system (1) is the sequence denoted $x\left(k, x_{0}\right), k \in \mathbb{N}$.

Next, the trajectories of the dynamical systems (1) will be characterized from the point of view of the sublevel sets of given function defined over the state space $\mathbb{R}^{n}$. For a given function $h \in \mathcal{C}^{0}\left(\mathbb{R}^{n}, \mathbb{R}^{m}\right)$, the sublevel set is defined as:

$$
\mathcal{L}^{h}=\left\{x \in \mathbb{R}^{n} \mid h(x) \leq 0\right\}
$$

At each time instant $k \in \mathbb{N}$, the state $x\left(k, x_{0}\right)$ can be characterised by the set membership with respect to a given sublevel set $\mathcal{L}^{h}$. The next definition extends this set membership to a complete trajectory.

Definition II.1. Given the initial condition $x_{0}$ of the system (1) and a function $h \in \mathcal{C}^{0}\left(\mathbb{R}^{n}, \mathbb{R}\right)$, we denote the Validation Index Set (VIS) as the maximal ordered collection of indices $\mathcal{T} \subset \mathbb{N}$ such that $x\left(k, x_{0}\right) \in \mathcal{L}^{h}$ for any $k \in \mathcal{T}$.

Obviously, the VIS is dependent on the initial conditions and the topology of the sublevel-set. In order to formally describe this dependence in mathematical terms let us introduce the functional:

$$
\begin{aligned}
\mathcal{T}: \quad \mathbb{R}^{n} \times \mathcal{C}^{0}\left(\mathbb{R}^{n}, \mathbb{R}\right) & \longrightarrow 2^{\mathbb{N}} \\
(x, h) & \longmapsto\left\{t \in \mathbb{N} \mid f^{t}(x) \in \mathcal{L}^{h}\right\}
\end{aligned}
$$

Example II.1. Consider the autonomous nonlinear system :

$$
x_{k+1}=\left[\begin{array}{c}
0.8 x_{1} \\
0.8 x_{1}^{3}+0.5 x_{2}^{2}-1
\end{array}\right]
$$

and the circular paraboloid $h:\left(x_{1}, x_{2}\right) \mapsto x_{1}^{2}+x_{2}^{2}-0.85^{2}$. Then $\mathcal{L}_{0}^{h}$ corresponds to a circle with a radius of 0.85 . A trajectory of the system is depicted in Fig. 1 for the initial state $x_{0}=\left[\begin{array}{ll}-1 & 1\end{array}\right]^{\top}$ leading to $\mathcal{T}\left(x_{0}, h\right)=\{2,4,5,6, \ldots\}$.

\section{B. Weak satisfaction of constraints}

Definition II.2 (Weak constraint satisfaction). Given $p \in \mathbb{N}$, the trajectory of the system (1) is weakly p-satisfying the constraint $h(x) \leq 0$ if it exists a function $r: \mathbb{N} \rightarrow \mathbb{N}_{[1, p]}$ such that $x\left(k+r(k), x_{0}\right) \in \mathcal{L}^{h}$ for any $k \in \mathbb{N}$.

In short, the weak $p$-satisfaction of a constraint indicates that a violation of the inequality will last for at most $p$ timeinstants along the trajectory. Is worth to be mention that whenever a trajectory is weakly $p$-satisfying a constraint, it will be satisfying also for any index grater than $p$. Thus the subsequent developments we will be interested in the minimal index of weak constraint satisfaction.

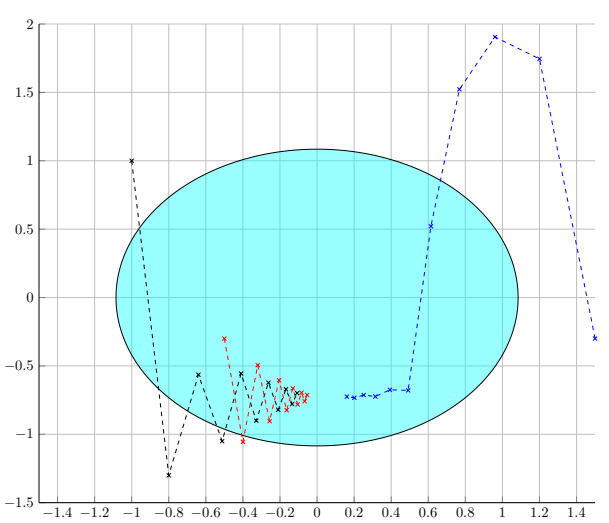

Fig. 1. Trajectory of the system (4)

Theorem II.1. There exists $p \in \mathbb{N}$ such that the constraint $h(x) \leq 0$ is weakly $p$-satisfied by the trajectory of the system (1) with initial condition $x_{0} \in \mathbb{R}^{n}$ if and only if

- $\mathcal{T}\left(x_{0}, h\right)$ is unbounded;

- The optimization problem:

$$
\begin{gathered}
\max _{j \in \mathbb{N}}\left\{t_{j+1}-t_{j}\right\} \\
\text { with } t_{j} \in \mathcal{T}\left(x_{0}, h\right)
\end{gathered}
$$

has a bounded solution.

Proof. If $h(x)$ is weakly $p$-satisfied for $x\left(k, x_{0}\right) \forall k \in \mathbb{N}$ then there exists a function $r: \mathbb{N} \rightarrow \mathbb{N}_{[1, p]}$ such that

$$
x\left(k+r(k), x_{0}\right) \in \mathcal{L}^{h} \forall k \in \mathbb{N}
$$

and the Validation Index Set verifies:

$$
\{r(0), r(1)+1, \ldots, r(k)+k, \ldots\} \subset \mathcal{T}\left(x_{0}, h\right)
$$

Consequently $\mathcal{T}\left(x_{0}, h\right)$ is unbounded and the optimization problem is feasible (any element $t_{j} \in \mathcal{T}\left(x_{0}, h\right)$ has a successor $\left.t_{j+1}\right)$. Since $\mathcal{T}\left(x_{0}, h\right)$ is an ordered subset of $\mathbb{N}$ it is countable and the difference $t_{j+1}-t_{j} \leq p$ using the boundedness of $r($.$) .$

If $\mathcal{T}\left(x_{0}, h\right)=\left(t_{i}\right)_{i \in \mathbb{N}}$ is (ordered) unbounded set and the optimization problem has a bounded solution $p$, then if we define the function :

$$
\begin{aligned}
r: \mathbb{N} & \longrightarrow \mathbb{N}_{[1, p]} \\
k & \longmapsto t_{k}-k
\end{aligned}
$$

the trajectory $x\left(k, x_{0}\right) \quad k \in \mathbb{N}$ is weakly $p$-satisfying the constraint according to Definition II.2.

When dealing with vector functions, the weak $p$ satisfaction of constraints will be considered by evaluating the range of violation for each of the components, taken independently. More precisely, given the vector function $h=\left[h_{1}, \ldots, h_{m}\right]^{T} \in \mathcal{C}^{0}\left(\mathbb{R}^{n}, \mathbb{R}^{m}\right)$ constructed using the element-wise constraints $h_{i}(x) \leq 0$, the following definition will be used. 
Definition II.3 (Weak vector-constraint satisfaction). The trajectory of the system (1) with initial condition $x_{0} \in \mathbb{R}^{n}$ is weakly p-satisfying the vector constraints $h(x) \leq 0$ if it exists a function $r: \mathbb{N} \rightarrow \mathbb{N}_{[1, p]}^{m}$ such that

$$
x\left(k+r_{i}(k), x_{0}\right) \in \mathcal{L}^{h_{i}}, \forall i \in\{1, \ldots, m\}
$$

and for any $k \in \mathbb{N}$.

Theorem II.2. The $m$-dimensional vector-constraint $h(x) \leq 0$ is weakly satisfied by the trajectory of the system (1) with initial condition $x_{0} \in \mathbb{R}^{n}$ if and only if

- The sets $\mathcal{T}\left(x_{0}, h_{i}\right)$ with $i \in \mathbb{N}_{[1, m]}$ are unbounded;

- The optimization problem:

$$
\begin{gathered}
\max _{i \in \mathbb{N}_{[1, m]}} \max _{j \in \mathbb{N}}\left\{t_{j+1}-t_{j}\right\} \\
\text { with } t_{j} \in \mathcal{T}\left(x_{0}, h_{i}\right)
\end{gathered}
$$

has a bounded solution.

Proof. According to Theorem II.1 applied here for each $i \in$ $\mathbb{N}_{[1, m]}$, the constraint $h_{i}(x) \leq 0$ is weakly satisfied by the trajectory if and only if the optimization problem (5) has bounded solution.

As a consequence, the optimization (6) is feasible and has a bounded solution given by the most conservative index of weak satisfaction.

At the level of trajectories, the weak satisfaction of the constraints doesn't guarantee the simultaneous satisfaction of constraints on a finite time interval as illustrated next.

Example II.2. Consider the functions:

$$
\begin{aligned}
& h_{1}\left(x_{1}, x_{2}\right)=4\left(x_{1}-1\right)^{2}+\left(x_{2}-3\right)^{2}-16 \\
& h_{2}\left(x_{1}, x_{2}\right)=4\left(x_{1}-1\right)^{2}+\left(x_{2}+3\right)^{2}-16
\end{aligned}
$$

and the dynamics:

$$
x(k+1)=\left[\begin{array}{cc}
0.9 & 0 \\
0 & -1
\end{array}\right] x(k)
$$

The first constraint is weakly 3-satisfied while the second is weakly 2-satisfied by the trajectory initiated in $x_{0}=$ $\left[\begin{array}{ll}2.8 & 0.8\end{array}\right]^{T}$. As it can be seen in the Fig. 2 the trajectory converge to a limit cycle which satisfies alternatively (but not simultaneously) both constraints and the difference in between the indices of weak satisfaction are related here to the choice of the initial conditions.

\section{Extensions to tubes of trajectories}

The definition of the VIS can be extended from trajectories to tubes of trajectories. The functional which associates to a set of initial conditions in $\mathbb{R}^{n}$ the time-indices which validate the sublevel set is defined as:

$$
\begin{aligned}
\mathcal{S}: \quad 2^{\mathbb{R}^{n}} \times \mathcal{F}\left(\mathbb{R}^{n}, \mathbb{R}\right) & \longrightarrow 2^{\mathbb{N}} \\
(X, h) & \longmapsto\left\{t \in \mathbb{N} \mid f^{t}(X) \subset \mathcal{L}^{h}\right\}
\end{aligned}
$$

In other words, for a given subset $X$ of $\mathbb{R}^{n}$, the VIS $S(X, h)$ is an ordered subset of $\mathbb{N}$ which collects the indices of successive images of initial conditions in $X$ via $f($.

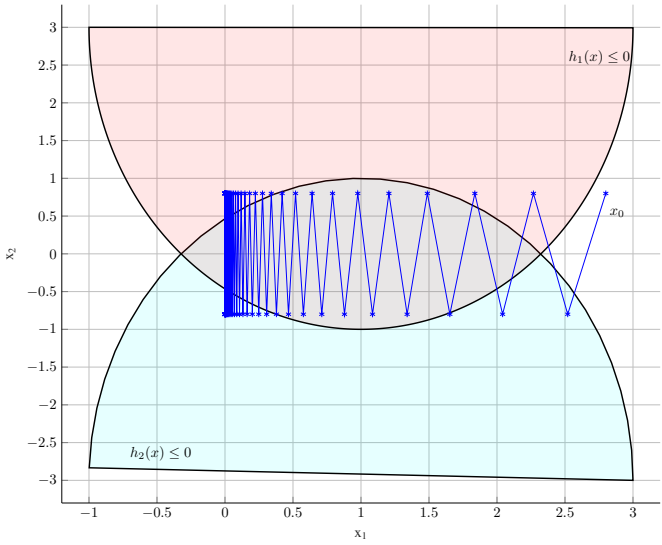

Fig. 2. Weak satisfaction of constraints (7)

such that the trajectories are simultaneously included in the sublevel set $\mathcal{L}^{h}$. The VIS for tubes of trajectories is the intersection of VIS for each individual trajectories within the tube:

$$
\mathcal{S}(X, h)=\bigcap_{x \in X} \mathcal{T}(x, h)
$$

Theorem II.3. The tube of trajectories of the system (1) initiated in $X \subset \mathbb{R}^{n}$ is weakly $p$-satisfying the $m$-dimensional vector-constraint $h(x) \leq 0$ if and only if

- The sets $\mathcal{S}\left(X, h_{i}\right)$ with $i \in \mathbb{N}_{[1, m]}$ are unbounded;

- The optimization problem:

$$
\begin{gathered}
p=\max _{i \in \mathbb{N}_{[1, m]}} \max _{j \in \mathbb{N}}\left\{t_{j+1}-t_{j}\right\} \\
\text { such that } t_{j} \in \mathcal{S}\left(X, h_{i}\right)
\end{gathered}
$$

has a bounded solution.

Proof. If there exist an unboundedness of the set $\mathcal{S}\left(X, h_{i}\right)$ ensures the trajectories validate regularly the constraint and thus each index has a successor in this ordered set. Conversely, the weak constraint satisfaction guarantees the finite distance in between two indices of validation in forward time and thus unboundedness of $\mathcal{S}\left(X, h_{i}\right)$.

The second condition translates in the mathematical form the alternative of choices: either the two successive states are validating the constraints or the validation will be enforced after exactly $p$ iterations.

The previous concepts are useful in constrained control, but there is one particular problem of interest: the case of initial conditions given by the constraint set itself. Let the intersection of the sublevel set of the vector function $h \in$ $\mathcal{C}^{1}\left(\mathbb{R}^{n}, \mathbb{R}^{m}\right)$ :

$$
\Omega(h)=\left\{x \in \mathbb{R}^{n} \mid h(x) \leq 0\right\} .
$$

Definition II.4 (Weak satisfaction of self-constraints). The tube of trajectory of the system (1) initiated in $\Omega(h)$ is weakly p-satisfying the constraints $h(x) \leq 0$ if it exists a function $r: \mathbb{N} \rightarrow \mathbb{N}_{[1, p]}^{m}$ such that

$$
x\left(k+r_{i}(k), x_{0}\right) \in \mathcal{L}^{h_{i}}, \forall k \in \mathbb{N}
$$


and for all $i \in\{1, \ldots, m\}$ where $m$ is the dimension of the vector function $h($.$) .$

Certifying the weak satisfaction of a set of constraints is a direct application of the Theorem II.3. If it exists, the minimal index is is the solution of the optimization:

$$
\begin{gathered}
p=\max _{i \in \mathbb{N}_{[1, m]}} \max _{j \in \mathbb{N}}\left\{t_{j+1}-t_{j}\right\} \\
\text { with } t_{j} \in \mathcal{S}\left(\Omega(h), h_{i}\right)
\end{gathered}
$$

It should be noticed that in the general case the selfconstraint satisfaction is not necessarily implying the inclusion of the images of the set $\Omega(h)$ into itself (see the tube of trajectories corresponding to the Example II.2).

\section{WEAK SET INVARIANCE}

\section{A. The general framework}

The previous section treated the satisfaction of constraints at the level of inequalities and their sublevel set, the vector functions were treated also in terms of validation of element-wise constraints. This constraint satisfaction was not enforced simultaneously at some time-instants along a trajectory or tube of trajectories. In this section, we concentrate on the set-membership of the states along the trajectories of dynamical systems. In particular we aim to characterize the properties of the trajectories that leave the set $\Omega$ for finite intervals before coming back. This notion is closely related to the positive invariance as described by the next definition.

Definition III.1 (Weak invariance). Let $p \in \mathbb{N}$. The set $\Omega \subset$ $\mathbb{R}^{n}$ is weakly p-invariant with respect to the system (1) if for any $x_{0} \in \Omega$, it exists a function $r: \mathbb{N} \rightarrow \mathbb{N}_{[1, p]}$ such that $x\left(k+r(k), x_{0}\right) \in \Omega$ for any $k \in \mathbb{N}$.

Lemma III.1. A set $\Omega \subset \mathbb{R}^{n}$ containing the origin in its interior is weakly p-invariant with respect to the system (1) if the constrained $\mu^{\Omega}(x) \leq 0$ defined using

$$
\mu^{\Omega}(x)=\{\min \alpha \in[-1, \infty): x \in(1+\alpha) \Omega\},
$$

is weakly p-satisfied by the tube of trajectories of the system (1) initiated in $\Omega$.

Proof. According to the Definition III.1, a link needs to be established between the set-membership and the sublevelset of a given inequality. The statement of the Theorem establishes this link under the assumption that the origin is an interior point of $\Omega$ by means of a Minkowski functional (15). Indeed, it can be easily verified that $\mathcal{L}^{\mu^{\Omega}}=\Omega$. The proof is complete by noting that according to Definition II.2, the weak satisfaction of the constraint $\mu^{\Omega}(x) \leq 0$ guarantees the existence of a function $r: \mathbb{N} \rightarrow \mathbb{N}_{[1, p]}$ such that

$$
x\left(k+r(k), x_{0}\right) \in \mathcal{L}^{\mu^{\Omega}}=\Omega .
$$

Theorem III.2. A set $\Omega \subset \mathbb{R}^{n}$ containing the origin in its interior is weakly p-invariant with respect to the system (1) if and only if

- $\mathcal{T}\left(x_{0}, \mu^{\Omega}\right)$ is unbounded for all $x_{0} \in \Omega$;
- The optimization problem:

$$
\begin{gathered}
\max _{x_{0} \in \Omega} \max _{j \in \mathbb{N}}\left\{t_{j+1}-t_{j}\right\} \\
\text { with } t_{j} \in \mathcal{T}\left(x_{0}, \mu^{\Omega}\right)
\end{gathered}
$$

has a bounded solution.

Proof. First if the set is weakly $p$-invariant then for each index $k$ in $\mathcal{T}\left(x_{0}, \mu^{\Omega}\right)$ it exists an index $k+r(k) \in \mathcal{T}\left(x_{0}, \mu^{\Omega}\right)$ and thus recursively there exists a successor index thus proving the set is unbounded. Consequently the optimization problem is feasible and upper bounded by the index $p$ of weak invariance. Thus the optimum exists.

Conversely, the satisfaction of the first conditions proves the feasibility of the optimization problem. By choosing $p$ the optimal solution, the conditions of weak invariance are fulfilled.

B. Weak invariance of polyhedral set with respect to LTI dynamics

As shown in the case of weak constraint satisfaction, whenever the dynamical system and the set of constraints has additional structural properties, the computation of the indices of weak invariance can be enhanced. The ultimate objective of the present section is to present a commonly encountered case for which the computation is finitely determined.

Next we will detail the case of linear time-invariant systems:

$$
x(k+1)=A x(k) .
$$

with a Schur matrix $A$.

Lemma III.3. Consider a bounded polyhedral set $\Omega \subset \mathbb{R}^{n}$ containing the origin in its interior. Let $p$ be the minimal index such that $\Omega$ is weakly $p$-invariant with respect to the asymptotically stable system (17). Then $p$ is the solution of the optimization problem:

$$
\begin{gathered}
\max _{x_{0} \in \bar{\Omega}} \max _{j \in \mathbb{N}}\left\{t_{j+1}-t_{j}\right\} \\
\text { with } t_{j} \in \mathcal{T}\left(x_{0}, \mu^{\Omega}\right)
\end{gathered}
$$

Proof. The Lemma claims that the index of weak invariance can be computed by optimizing over the constraint satisfaction indices of the trajectories initiated on $\bar{\Omega}$. By contradiction, suppose that the maximum index corresponds exclusively to an interior point $\tilde{x}$ such that $x(0, \tilde{x}) \in \Omega$ and $x(p, \tilde{x}) \in \Omega$ but $x(i, \tilde{x}) \notin \Omega$ for all $i \in \mathbb{N}_{[1, p-1]}$.

Note however that there exist $\gamma>1$ such that $\gamma \tilde{x} \in \bar{\Omega}$. By linearity of the dynamics and convexity of the set $\Omega$ it follows $\gamma x(i, \tilde{x}) \notin \Omega$ for all $i \in \mathbb{N}_{[1, p-1]}$. The constraint satisfaction index for the set $\Omega$ being $p$ leads to

$$
\gamma x(p, \tilde{x})=x(p, \gamma \tilde{x}) \in \Omega
$$

which shows that the optimum value of the optimization is obtained by the point $\gamma \tilde{x} \in \bar{\Omega}$ which leads to a contradiction.

Theorem III.4. Consider a bounded polyhedral set $\Omega \subset \mathbb{R}^{n}$ containing the origin in its interior. The minimal index 
$p$ such that $\Omega$ is weakly $p$-invariant with respect to the asymptotically stable system (17) is finitely determined.

Proof. Lemma III.3 indicates the computation of the index $p$ can be resumed to the study of the frontier of the set $\Omega$. Given the asymptotic stability property of the dynamics and the origin as an interior point of $\Omega$, there exist a finite time instant $\bar{p}$ such that

$$
\mathbb{N}_{[\bar{p}, \infty)} \subset \mathcal{T}\left(v_{i}, \mu^{\Omega}\right)
$$

for all $v_{i} \in \mathcal{V}(\Omega)$. Exploiting the existence of this finite upper bound and the finite number of vertices for a bounded polyhedral set, the finite computation of the index $p$ follows.

Example III.1. Consider a bounded polyhedron $X_{0}$ and the linear system :

$$
x(k+1)=\left[\begin{array}{cc}
0.9806 & 0.0685 \\
-0.4842 & 0.7134
\end{array}\right] x(k)
$$

The successive images of $X_{0}$ are depicted in Fig. 3 with the validation indices of trajectories initiated in $X_{0}$ with respect to each halfspace constituting $X_{0}$ itself. In this example the minimal index of weak invariance of $X_{0}$ is $p=12$ which is the maximal gap between two successive elements of $\mathcal{T}\left(v_{i}, \mu^{X_{0}}\right)$ where $v_{i} \in \mathcal{V}\left(X_{0}\right)$.

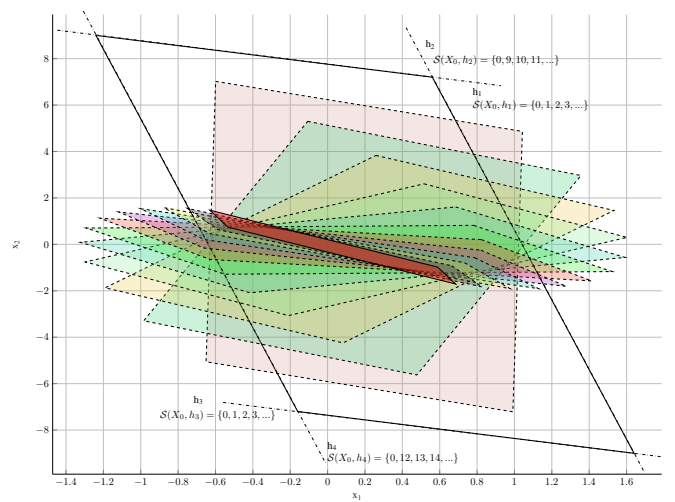

Fig. 3. A weak invariant set and the successive images of $X_{0}$ through the system (19). For completeness the indices of weak constraints satisfaction is presented for each halfspace involved in the polyhedral set description.

\section{STRONG CONSTRAINT SATISFACTION AND STRONG INVARIANCE}

The weak constraint satisfaction and weak invariance offer two different perspectives on the validation of static constraints along the trajectories of a dynamical system. The present section aims to link the two notions through a strong version which imposes additional restrictions on the allowed interval between violation of constraints.

Definition IV.1 (Strong vector-constraint satisfaction). The tube of trajectories of the system (1) with initialized in $X \subset$
$\mathbb{R}^{n}$ is strongly $p$-satisfying the vector constraints $h(x) \leq 0$ with $h: \mathbb{R}^{n} \rightarrow \mathbb{R}^{m}$ if

$$
\left.\begin{array}{c}
x\left(k, x_{0}\right) \in \mathcal{L}^{h_{i}} \\
x\left(k+1, x_{0}\right) \notin \mathcal{L}^{h_{i}}
\end{array}\right\} \Longrightarrow x\left(k+p, x_{0}\right) \in \mathcal{L}^{h_{i}},
$$

for each $i \in \mathbb{N}_{[1, m]}$, for any $x_{0} \in X$ and for any $k \in \mathbb{N}$.

Theorem IV.1. The $m$-dimensional vector-constraint $h(x) \leq 0$ is strongly $p$-satisfied by the trajectory of the system (1) with initial condition $x_{0} \in X \subset \mathbb{R}^{n}$ if and only if

- The sets $\mathcal{T}\left(x_{0}, h_{i}\right)$ with $i \in \mathbb{N}_{[1, m]}$ are unbounded;

- For each $i \in \mathbb{N}_{[1, m]}$ and for any successive elements $t_{j}, t_{j+1} \in \mathcal{T}\left(x_{0}, h_{i}\right)$ we have either $t_{j+1}-t_{j}=1$ or $t_{j}+p \in \mathcal{T}\left(x_{0}, h_{i}\right)$.

Without establishing a formal result, it can be noticed that strong constraint satisfaction index can (and usually is) larger than the weak counterpart. Also, from the computational point of view, the case of stable linear systems leads to a finite determination of the strong index of constraints' satisfaction.

Corollary IV.2. If the $m$-dimensional vector-constraint $h(x) \leq 0$ is strongly $p$-satisfied by the trajectory of the system (1) with initial condition $x_{0} \in X \subset \mathbb{R}^{n}$, then the same vector constraints are weakly $p$-satisfied.

Proof. By simply choosing the function:

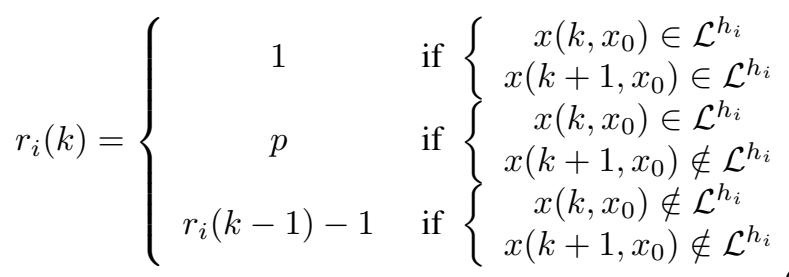

Definition IV.2 (Strong invariance). Let $p \in \mathbb{N}$. The set $\Omega \subset$ $\mathbb{R}^{n}$ is strongly p-invariant with respect to the system (1) if

$$
\left.\begin{array}{c}
x\left(k, x_{0}\right) \in \Omega \\
x\left(k+1, x_{0}\right) \notin \Omega
\end{array}\right\} \Longrightarrow x\left(k+p, x_{0}\right) \in \Omega,
$$

for each $x_{0} \in \Omega$, and for all $k \in \mathbb{N}$.

Theorem IV.3. A set $\Omega \subset \mathbb{R}^{n}$ containing the origin in its interior is strongly p-invariant with respect to the system (1) if and only if

- $\mathcal{T}\left(x_{0}, \mu^{\Omega}\right)$ is unbounded for all $x_{0} \in \Omega$;

- For any successive elements $t_{j}, t_{j+1} \in \mathcal{T}\left(x_{0}, \mu^{\Omega}\right)$ we have either $t_{j+1}-t_{j}=1$ or $t_{j}+p \in \mathcal{T}\left(x_{0}, h_{i}\right)$.

The proof is omitted for space reasons its argument following the same argument as the proof of Theorem IV.1.

Corollary IV.4. If the set $\Omega \subset \mathbb{R}^{n}$ is strongly p-invariant with respect to the system (1) then it is weakly p-invariant.

Note also that strong invariance index is grater or equal with the weak invariance index. Next result bridges the gap between the strong $p$-satisfaction of (self)-constraints and the strong positive $p$-invariance. 


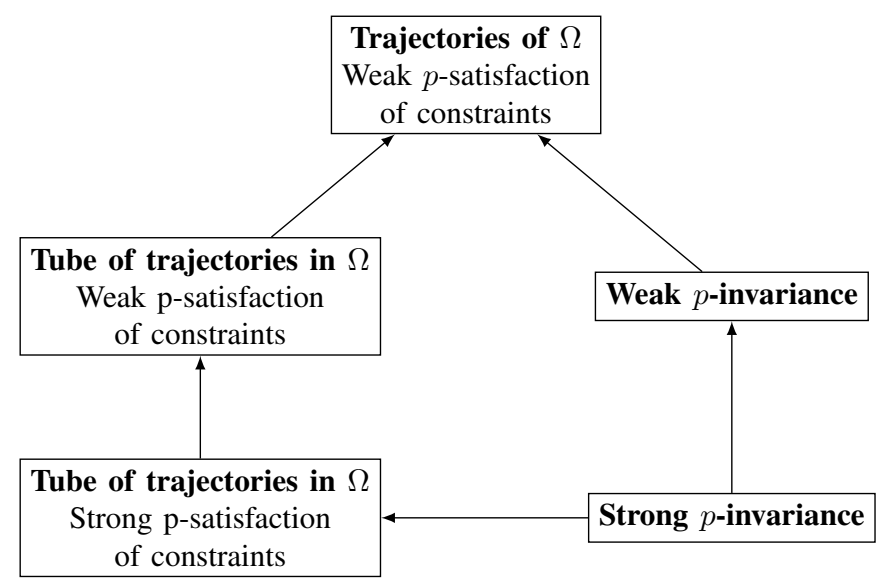

Fig. 4. Summary of concepts introduced with the structural relationship between the constraints satisfaction and the invariance and subsequently on the qualitative assessment of these properties.

Theorem IV.5. Let a set $\Omega=\left\{x \in \mathbb{R}^{n} \mid h_{i}(x) \leq 0\right\}$ containing the origin in its interior. If the set is strongly $p$-invariant with respect to the dynamics (1) then the trajectory initiated in $\Omega$ are strongly $p$-satisfying the vector-constraints defining this set.

Proof. The level set of the Minkowski function satisfies:

$$
\mathcal{L}^{\mu^{\Omega}}=\bigcap_{i} \mathcal{L}^{h_{i}}
$$

and ensures:

$$
\mu^{\Omega}\left(f^{p}(\Omega)\right) \leq 0 \Longrightarrow h_{i}\left(f^{p}(\Omega)\right) \leq 0, \forall i \in \mathbb{N}_{[1, m]}
$$

Note that the assumption of the origin being an interior point can be relaxed by an appropriate change of coordinates and has been adopted here to facilitate the proof.

The main concepts presented in the paper are summarized together with their relationships in Figure 4.

The last results are recalling the computational aspects and underline the fact that in the case of linear systems the numerical tools for certifying the $p$-invariance are relatively mature and scale adequately with respect to the dimension of the state space or the complexity of the sets to be analysed.

Proposition IV.1. The strong $p$-invariance of a bounded polyhedral set, containing the origin in its interior, with respect to a stable linear dynamics (17) is equivalent with the strong $p$-satisfaction of the constraint $\mu^{\Omega}\left(v_{i}\right) \leq 0$ for $v_{i} \in \mathcal{V}(\Omega)$.

\section{CONCLUSION}

The paper revisited the notions of constraint satisfaction and positive invariance by introducing a relaxation (generalization) on the window on possible violations. If the classical notions impose a satisfaction of the set of constraints (or, alternatively, of the set-membership) at each sampling time, in the present work, the validation needs to be done at least once in a given time interval. The length of this time interval is parameterizing the weak or strong versions of the constraint satisfaction (invariance).

Is interesting to note that the classical rigid notions of positive invariance are covered by the present framework and additionally, the results are providing a link to alternative constructions in the literature as for example the periodic invariant sets.

Given the present foundation, the directions to be explored are related to

- the qualitative analysis of the link in between the length of the interval of constraint satisfaction (invariance) and the complexity of the set of constraints

- the extension from autonomous systems to (constrained) controlled systems

- the formulation of a robust version, to cope with the presence of bounded uncertainties.

\section{REFERENCES}

[1] J.-P. Aubin, Viability theory. Springer Science \& Business Media, 2009.

[2] F. Blanchini and S. Miani, Set-theoretic methods in control. Springer, 2008.

[3] D. Q. Mayne, J. B. Rawlings, C. V. Rao, and P. O. Scokaert, "Constrained model predictive control: Stability and optimality," Automatica, vol. 36, no. 6, pp. 789-814, 2000.

[4] E. G. Gilbert and I. Kolmanovsky, "Discrete-time reference governors for systems with state and control constraints and disturbance inputs," in Proceedings of 1995 34th IEEE Conference on Decision and Control, vol. 2. IEEE, 1995, pp. 1189-1194.

[5] M. M. Seron, X. W. Zhuo, J. A. De Doná, and J. J. Martínez, "Multisensor switching control strategy with fault tolerance guarantees," Automatica, vol. 44, no. 1, pp. 88-97, 2008.

[6] G. Bitsoris, "Positively invariant polyhedral sets of discrete-time linear systems," International Journal of Control, vol. 47, no. 6, pp. 1713$1726,1988$.

[7] C. E. T. Dorea and J. Hennet, "(a, b)-invariant polyhedral sets of linear discrete-time systems," Journal of Optimization Theory and Applications, vol. 103, no. 3, pp. 521-542, 1999.

[8] F. Blanchini, "Set invariance in control," Automatica, vol. 35, no. 11, pp. 1747-1767, 1999.

[9] G. Bitsoris and L. Truffet, "Positive invariance, monotonicity and comparison of nonlinear systems," Systems \& control letters, vol. 60, no. 12 , pp. 960-966, 2011.

[10] E. G. Gilbert and K. T. Tan, "Linear systems with state and control constraints: The theory and application of maximal output admissible sets," IEEE Transactions on Automatic control, vol. 36, no. 9, pp. 1008-1020, 1991.

[11] F. Scibilia, S. Olaru, and M. Hovd, "On feasible sets for mpc and their approximations," Automatica, vol. 47, no. 1, pp. 133-139, 2011.

[12] Y. I. Lee and B. Kouvaritakis, "Constrained robust model predictive control based on periodic invariance," Automatica, vol. 42, no. 12, pp. 2175-2181, 2006.

[13] S. Scialanga, "Reduced-complexity interpolating control of constrained interconnected systems and applications," Ph.D. dissertation, University of Glasgow, 2020.

[14] M. Soyer, S. Olaru, and Z. Fang, "A novel Motion Cueing Algorithm based on real-time optimization and periodic invariant sets," in 4th IEEE Conference on Control Technology and Applications (CCTA 2020), Montréal, Canada, Aug. 2020. [Online]. Available: https://hal-centralesupelec.archives-ouvertes.fr/hal-02899834 